\title{
A LITERATURE SURVEY ON VARIOUS ILLUMINATION NORMALIZATION TECHNIQUES FOR FACE RECOGNITION WITH FUZZY K NEAREST NEIGHBOUR CLASSIFIER
}

\author{
A. Thamizharasi ${ }^{1}$ and J.S. Jayasudha ${ }^{2}$ \\ ${ }^{I}$ Department of Computer Science and Engineering, Mohandas College of Engineering \& Technology, India \\ E-mail: radhatamil1@ @rediffmail.com \\ ${ }^{2}$ Department of Computer Science and Engineering, SCT College of Engineering, India \\ E-mail: jayasudhajs@gmail.com
}

\begin{abstract}
The face recognition is popular in video surveillance, social networks and criminal identifications nowadays. The performance of face recognition would be affected by variations in illumination, pose, aging and partial occlusion of face by Wearing Hats, scarves and glasses etc. The illumination variations are still the challenging problem in face recognition. The aim is to compare the various illumination normalization techniques. The illumination normalization techniques include: Log transformations, Power Law transformations, Histogram equalization, Adaptive histogram equalization, Contrast stretching, Retinex, Multi scale Retinex, Difference of Gaussian, DCT, DCT Normalization, DWT, Gradient face, Self Quotient, Multi scale Self Quotient and Homomorphic filter. The proposed work consists of three steps. First step is to preprocess the face image with the above illumination normalization techniques; second step is to create the train and test database from the preprocessed face images and third step is to recognize the face images using Fuzzy $K$ nearest neighbor classifier. The face recognition accuracy of all preprocessing techniques is compared using the AR face database of color images.
\end{abstract}

Keywords:

Illumination Normalization, Contrast Stretching, Power Law, Homomorphic Filter, Log Transformations, FKNN Classifier

\section{INTRODUCTION}

Face recognition can be applied for a wide variety of problems like image and film processing, human-computer interaction, criminal identification etc. This has motivated researchers to develop computational models to identify the faces, which are relatively simple and easy to implement. A face image has high dimension.

\subsection{IMPORTANCE OF ILLUMINATION NORMALIZATION}

Illumination is considered one of the most difficult tasks for face recognition. Variations caused by pose, expression, occlusion or illumination is highly nonlinear, and making the detection task extremely complex [1]. Here, the illumination normalization is addressed in particular. Illumination is a very important problem in face recognition. Research has shown that for a face image, the variability caused by illumination changes even exceeds the variability caused by identity changes [2]. As an example, Fig.1 shows the face images of the same subject under two different illuminations from Yale Face Database.

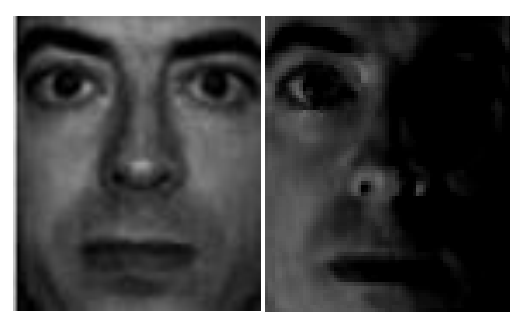

Fig.1. Examples of the same subject seen under different illuminations.

Such illuminations will largely influence the performance of face recognition. The techniques applied are

- Log transformations

- Power Law transformations

- Contrast stretching

- Histogram equalization

- Adaptive histogram equalization

- Homomorphic filter

- Single Scale Retinex

- Multiscale Retinex

- Difference of Gaussian

- DCT Normalization

- Gradientface

- Self-Quotient

- Multiscale Self Quotient

- DCT

- DWT

\subsection{ALGORITHM FOR FACE RECOGNITION USING ILLUMINATION NORMALIZATION TECHNIQUES}

The face recognition of the proposed method has the following steps by first creating the training and test database using AR face database:

Step 1: Preprocess the face images using any one of the illumination normalization techniques

Step 2: Repeat the step 1 for all images in the train database and the test database 
Step 3: Use the Fuzzy K nearest neighbour classifier to classify the images in the test database to the closest class by comparing it with train database.

Step 4: Find the face recognition accuracy rate i.e. the number of correct matches for the test in $\%$.

Face recognition accuracy $($ in $\%)=($ correct matches/Total number of tests) $* 100$

\section{ILLUMINATION TECHNIQUES}

\section{NORMALIZATION}

\subsection{LOG TRANSFORMATIONS}

The general form of the $\log$ transformation is

$$
\mathrm{s}=\operatorname{clog}\left(1+256^{*} \mathrm{r}\right)
$$

where, $r$ is the input image and it is assumed that $r \geq 0$, $s$ is the enhanced image and $\mathrm{c}$ is a constant which is equal to 1 . The log transformation maps a narrow range of low gray level values in the input image into a wider range of output values. The opposite is true of higher values of input levels. This log transformation could be used if we want to expand the valued of dark pixels in an image while compressing the higher-level values [4].

The Fig.2(a) shows the original image, Fig.2(b) shows the gray scale image and Fig.2(c) shows the log transformation applied image.

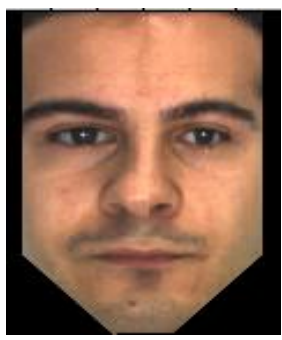

(a)

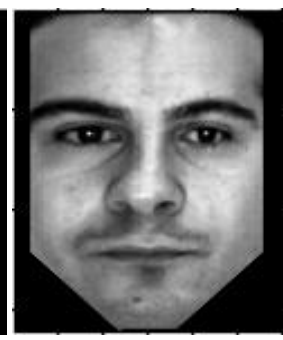

(b)

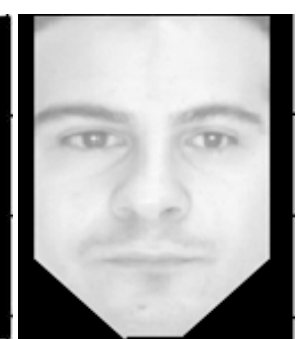

(c)
Fig.2(a). Original image (b). gray scale image (c). Log transformation applied image

\subsection{POWER LAW TRANSFORMATIONS}

Power-law transformations have the basic form

$$
S=c r^{\gamma}
$$

where, $c$ and $\gamma$ are positive constants. Here $\gamma=2$. As in the case of the log transformation, power-law curves with fractional values of $\gamma$ map a narrow range of dark input values into a wider range of output values, with the opposite being true for higher values of input pixels. A family of possible transformations curves obtained simply by varying $\gamma$. Curves with values of $\gamma>1$ have exactly the opposite effect as those generated with values of $\gamma<1$. The transformation curve reduces to identity transformation when $c=\gamma=1$. The exponent in the power-law equation is referred to as gamma. Hence this transformation is also called as gamma transformation. Gamma transformation is useful for contrast enhancement [4].

\subsection{CONTRAST STRETCHING}

One of the simplest piecewise linear functions is a contraststretching transformation. Low contrast images can result from poor illumination. The idea behind contrast stretching is to increase the dynamic range of the gray levels in the image being processed. The contrast enhanced image is given by the following equation,

$$
g=\frac{1}{1+\left(\frac{m}{f}\right)} r
$$

where, $\mathrm{g}$ is the contrast enhanced image, $\mathrm{m}$ is the mean of the input image, $f$ is the input image $r$ is the constant [4]. Here $\gamma=3$. The Fig.3(a) shows Power-law transformations applied image. Fig.3(b) shows contrast stretched images.

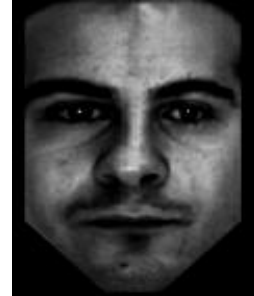

(a)

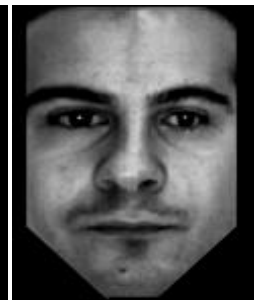

(b)
Fig.3(a). Power law image (b). Contrast stretched image

\subsection{HISTOGRAM EQUALIZATION}

Histogram Equalization is a method of contrast enhancement of an image using its histogram. If the pixel intensities in an image are lying in either dark or brighter side of histogram, then the histogram will have its peak values at different levels. The histogram equalization increases the contrast of image by uniformly distributing the intensities in entire range 0 to 255[4]. After histogram equalization, the histogram will have all its peak values at same level. Fig.4(a) shows the histogram equalized images.

\subsection{ADAPTIVEHIST EQUALIZATION}

ADAPTHISTEQ enhances the contrast of images by transforming the values in the intensity image I. Unlike HISTEQ, it operates on small data regions (tiles), rather than the entire image. Each tile's contrast is enhanced, so that the histogram of the output region approximately matches the specified histogram. It improves the local contrast of image. The Fig.4(b) shows the adaptive histogram equalized image.

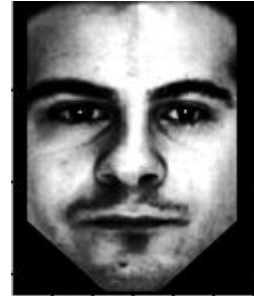

(a)

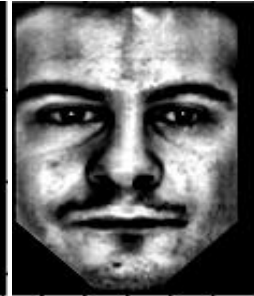

(b)
Fig.4(a). Histogram equalized image (b). Adaptive Histogram equalized image 


\subsection{HOMOMORPHIC FILTERS}

The Homomorphic filters are based on the well-known illumination-reflectance model [3].The Homomorphic filters controls the illumination and reflectance component and suitable for illumination normalization. An image $f(x, y)$ can be expressed as the product of the illumination $i(x, y)$, and the reflectance component $r(x, y)$ as follows:

$$
f(x, y)=i(x, y) \cdot r(x, y)
$$

This equation cannot be used to operate separately on the frequency components of illumination and reflectance directly because the Fourier transform of the product of the two functions is not separable.

The Illumination component of an image is generally characterized by the slow spatial variations while the reflectance components vary abruptly, particularly at the junctions of the dissimilar objects. These characteristics actually lead to associate the low frequencies of the Fourier transform of the logarithm of an image with illumination and the high frequencies with reflectance [15]. Fig.5(a) shows the homomorphic filtered image.

\subsection{SINGLE SCALE RETINEX (SSR)}

In 1971 Land and McCann introduce the idea that image $I(x$, $y)$ is the product of two components, illumination $L(x, y)$ and reflectance $R(x, y)$ [11].

$$
I(x, y)=L(x, y) R(x, y)
$$

Illumination contains geometric properties of the scene (i.e., the surface normal and the light source position) and reflectance contains information about the object. Based on the assumption that the illumination varies slowly across different locations of the image and the local reflectance may change rapidly across different location, the processed illumination should be drastically reduced due to the high-pass filtering, while the reflectance after this filtering should still be very close to the original reflectance.

The reflectance can be also finding by dividing the image by the low pass version if the original image, which is representing illumination components. Land proposed a technique called retinex, which is a combination of the words retina and cortex. It tries to explain model of the human visual system. The most interesting point for illumination normalization is the assumption that perception depends on the relative or surrounding illumination. It means that reflectance $R(x, y)$ equals the quotient of image $I(x, y)$ and the illumination $L(x, y)$ calculated by the neighbourhood of $I(x, y)$. It improves the visibility of dark object while maintaining the visual different of the light area.

Single scale retinex algorithm proposed by Jobson and Woodell [12] defines a Gaussian kernel to estimate the neighbourhood illumination. Additional the logarithmic transformation is employed to compress the dynamic range.

Reflectance image takes from the form:

$$
R_{S S R}(x, y)=\log I(x, y)-\log F(x, y) * I(x, y)
$$

where, * denotes the convolution operation and $F(x, y)$ is the surround Gaussian function. Fig.5(b) shows the Single Scale Retinex image.

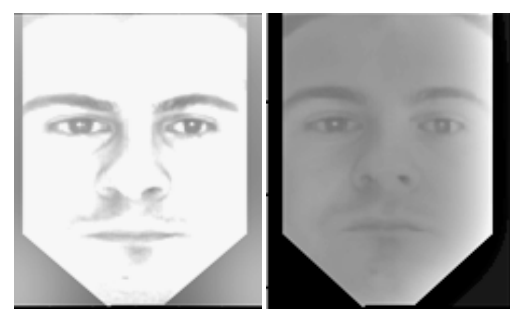

(a)

(b)

Fig.5(a). Homomorphic filtered image (b). Single Scale Retinex image

\subsection{MUTLI SCALE RETINEX (MSR)}

Rahman [13] improved previous method by estimating illumination as a combination of several weighting $\left(\omega_{\mathrm{n}}\right)$ Gaussian filters with different scales $(N)$. Reflectance image is defined by:

$$
R_{M S R}(x, y)=\sum_{n=1}^{N} \omega_{n}\{\log I(x, y)-\log [F(x, y) * I(x, y)]\}
$$

\subsection{THE DOG FILTERING - BASED NORMALIZATION TECHNIQUE}

(DOG) is a normalization technique which relies on the difference of Gaussians filter to produce the normalized image. Basically it applies a band pass filter to the input image and produces a normalized version of it [13]. Fig.6(a) shows the Multi Scale Retinex image and Fig.6(b) shows the DoG image.

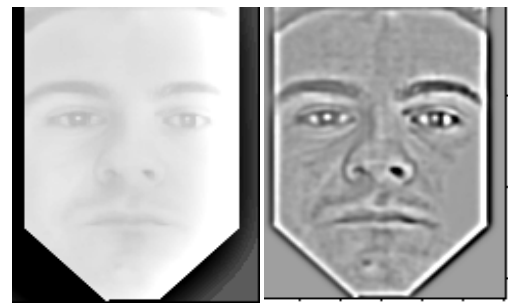

(a)

(b)

Fig.6(a). Multi Scale Retinex image (b). DoG image

\subsection{THE DCT BASED NORMALIZATION TECHNIQUE}

The DCT based normalization technique (DCT) was proposed by Chen et al. in [12]. The technique sets a number of DCT coefficients corresponding to low-frequencies to zero and hence tries to achieve illumination invariance. Here 100 DCT coefficients are set to zero. Fig.7(a) shows 100 DCT coefficients set to zero and fig.7(b) shows 10 DCT coefficients set to zero. Here DCT 10 coefficients are used for testing. 


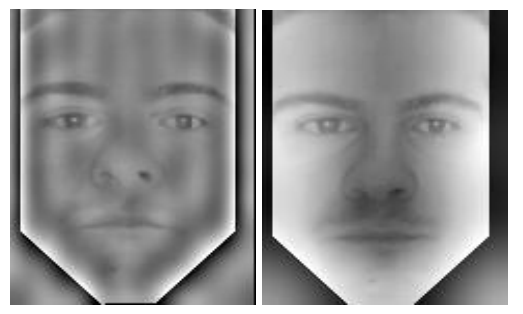

(a)

(b)

Fig.7(a). 100 DCT coefficients set to zero (b). 10 DCT coefficients set to zero.

\subsection{THE GRADIENT FACES NORMALIZATION TECHNIQUE}

The Gradient faces-based normalization technique (GRF) represents a normalization technique first proposed in [6]. The function computes the orientation of the image gradients in each pixel of the face images and uses the computed face representation as an illumination invariant version of the input image. Figure.8a shows the gradient face image.

\subsection{THE SINGLE SCALE SELF-QUOTIENT IMAGE}

The single scale self-quotient image (SSQ) was introduced to the field of face recognition by Wang et al. in [14]. The technique exhibits similarities to the single scale retinex technique, but unlike the SSR technique uses an anisotropic filter for the smoothing operation [10].Figure $8 \mathrm{~b}$ shows the selfquotient face image.

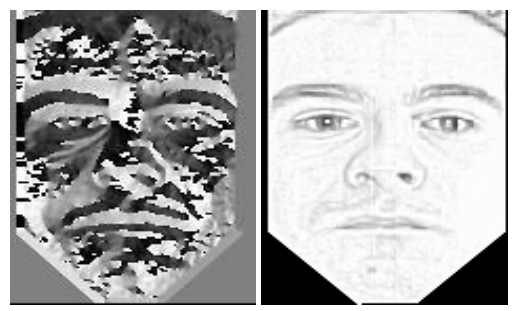

(a)

(b)

Fig.8(a). Gradient face image (b). Self Quotient image

\subsection{THE MULTI SCALE SELF-QUOTIENT IMAGE}

The multi scale self-Quotient image was also introduced to the field of face recognition by Wang et al [14].

$$
Q(x, y)=\sum_{k=1}^{n} m_{k} T\left\{Q_{k}(x, y)\right\}
$$

where, $m_{k}$ are weighting factors, $T$ is nonlinear function and $Q_{k}$ are quotient images corresponding to $k$ scale

$$
Q_{k}(x, y)=\frac{I(x, y)}{\left(\frac{1}{N} W_{k} G_{k}\right) * I(x, y)}, k=1, \ldots n
$$

where, $\mathrm{N}$ is normalization factor, $W_{k} G_{k}$ are weighted Gaussian kernels. The technique is complex and time consuming and hence the accuracy for this technique is not presented. Fig.9 shows the multi scale self-quotient face image.

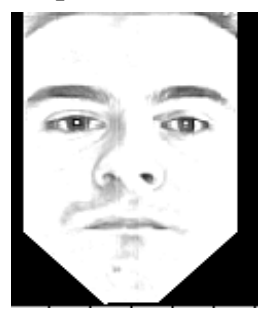

Fig.9. Multi scale Self Quotient image

\subsection{THE DISCRETE COSINE TRANSFORM}

The Discrete Cosine Transform DCT is often used in signal and image processing, especially for lossy compression, because it has a strong energy compaction property [17]. In DCT most of the signal information tends to be concentrated in a few lowfrequency components of the DCT.

DCT attempts to de-correlate the image data. After decorrelation each transform coefficient can be encoded independently without losing compression efficiency [17]. This section describes the DCT and some of its important properties.

\subsubsection{The Two-Dimensional DCT:}

The 2-D DCT is a direct extension of the 1-D case and is given by,

$$
\begin{aligned}
& C(u, v)= \\
& \alpha(u) \alpha(v) \sum_{x=0}^{N-1} \sum_{y=0}^{N-1} f(x, y) \cos \left[\frac{\pi(2 x+1) u}{2 N}\right] \cos \left[\frac{\pi(2 y+1) v}{2 N}\right]
\end{aligned}
$$

for $u, v, 0,1,2,, N-1$ and $\alpha(u)$ and $\alpha(v)$ are defined in equation of 1D DCT. The inverse transform is defined as,

$$
\begin{aligned}
& f(x, y)= \\
& \sum_{u=0}^{N-1} \sum_{v=0}^{N-1} \alpha(u) \alpha(v) C(u, v) \cos \left[\frac{\pi(2 x+1) u}{2 N}\right] \cos \left[\frac{\pi(2 y+1) v}{2 N}\right]
\end{aligned}
$$

for $x, y=0,1,2,, N-1$. The 2-D basis functions can be generated by multiplying the horizontally oriented $1-\mathrm{D}$ basis functions with vertically oriented set of the same functions [5]. Fig.10(a) shows the DCT image.

\subsection{DISCRETE WAVELET TRANSFORM}

The Discrete wavelet transform is a multi-resolution technique that analyses the signal in frequency domain and time domain simultaneously.

\subsubsection{D DWT:}

The 2D DWT is obtained by using 1D DWT. The onedimensional wavelet decomposition is first applied along the rows of the images, and then their results are further decomposed along the columns. This results in four decomposed sub-images L1, H1, V1, and D1.These sub-images represent different frequency localizations of the original image which refer to Low-Low, Low-High, High-Low and High-High respectively [16]. Their frequency components comprise the original frequency components but now in distinct ranges. In 
each iterative step, only the sub-image L1 is further decomposed.

In the 2D DWT, image of size $\mathrm{m} \times \mathrm{n}$ is divided into four sub bands L1, H1, V1, and D1 also called as approximation coefficients, Horizontal coefficients, vertical coefficients and Diagonal coefficients consists of sub image of size $\mathrm{m} / 2 \times \mathrm{n} / 2$.In this work, the approximation coefficients are chosen and $\mathrm{H} 1$, V1, and D1are omitted. Here the 2D DWT is applied at level 1 Fig.10(b) shows the DWT image

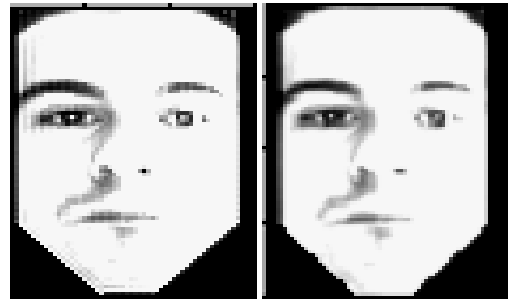

(a)

(b)

Fig.10(a). DCT image (b). DWT image

\section{FUZZY K NEAREST NEIGHBOUR CLASSIFIER}

In this paper, the classifier used for recognizing the image is Fuzzy K Nearest Neighbour Classifier. The algorithm is given below.

The fuzzy membership degree and each class center are gained through FKNN [7] algorithm. With FKNN algorithm, the computations of the membership degree can be realized through a sequence of steps:

Step 1: Compute the Euclidean distance matrix between pairs of feature vectors in training set.

Step 2: Set diagonal elements of this Euclidean distance matrix to infinity.

Step 3: Sort the distance matrix (treat each of its columns separately) in an ascending order. Collect the corresponding class labels of the patterns located in the closest neighborhood of the pattern under consideration (as we are concerned with " neighbors, this returns a list of " integers).

Step 4: Compute the membership degree to class ' $i$ ' for $j^{\text {th }}$ pattern using the expression proposed in the literature [8]

$$
u_{i j}=\left\{\begin{array}{c}
0.51+0.49 \times\left(n_{i j} / k\right) \\
0.49 \times\left(n_{i j} / k\right)
\end{array}\right.
$$

In the above expression $n_{i j}$ stands for the number of the neighbors of the data (pattern) that belong to the class. As usual, $u_{i j}$ satisfies two obvious properties:

$$
\begin{gathered}
\sum_{i=1}^{c} u_{i j}=1 \\
0<\sum_{j=1}^{N} u_{i j}<N
\end{gathered}
$$

Taking into account the fuzzy membership degree, the mean vector of each class is,

$$
\hat{m}_{i}=\frac{\sum_{j=1}^{N} u_{i j}^{\rho} x_{j}}{\sum_{j=1}^{M} u_{i j}^{\rho}}
$$

where, $\rho$ is a constant which controls the influence of fuzzy membership degree [7].

Therefore, the class center matrix $m$ and the fuzzy membership matrix $U$ can be achieved with the result of FKNN.

$$
\begin{gathered}
U=\left\lfloor u_{i j}\right\rfloor, i=1,2 \ldots c, j=1,2 \ldots N \\
m=\left[\widehat{m}_{i}\right], i=1,2 \ldots c
\end{gathered}
$$

In the above algorithm the preprocessed face image in test database is tested with preprocessed image in train database and the fuzzy membership is assigned to find the closest match for the given test image from the train database. The train image which has highest fuzzy membership value is chosen as the closest train image.

\section{IMPLEMENTATION}

\subsection{EXPERIMENTAL WORK}

The testing is done using the AR Database. The AR database (Martinez and Benavente, 1998, 2000) contains images of 100 persons taken in two different sessions. 50 are men and 50 are women [9]. In each session photos were taken in 13 different conditions. They are Neutral, expressions (anger, scream, and smile), illumination (right, left and both sides) and occlusions (eye occlusion, eye occlusion with left illumination, eye occlusion with right illumination, mouth occlusion, mouth occlusion with left illumination and mouth occlusion with right illumination).All the images are cropped to an image size of 160 $\times 120$ pixels.

There are 26 images for each person and totally there are 2600 images. AR face image subset is created with 14 images of each person with a total of 1400 images. This subset contains images of different illuminations and different expressions and neutral conditions.

Out of the 14 images of each person, the first image in neutral condition is used as train image. The remaining 13 images of each person with a total of 1300 images are used for testing. The face image used here is cropped at the jaw position automatically using the code written in MATLAB. Fig. 11 shows the sample face images in AR face database subset used for testing.

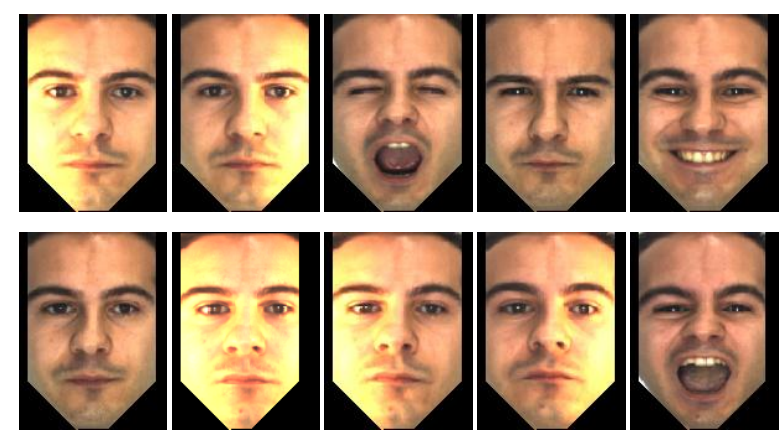




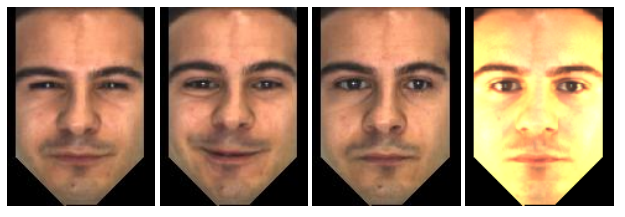

Fig.11. AR face subset database images

\section{RESULTS}

Face recognition is preprocessed with the above illumination normalization techniques are implemented in MATLAB 2013.The train database consists of 100 images and test database consists of 1300 images are created after preprocessing them.

The test image is classified using Fuzzy K nearest neighbour classifier to find the matching image. The face recognition accuracy $\%$ is calculated using the formula:

$\left(\begin{array}{l}\text { Face recognition } \\ \text { accuracy } \%\end{array}\right)=\left(\begin{array}{l}\text { No. of correct } \\ \text { matches }\end{array} / \begin{array}{l}\text { Total No. of } \\ \text { test images }\end{array}\right) * 100$

\subsection{ANALYSIS}

Face recognition is tested by using the test database with 1300 images and the train database with 100 images (1 train image per subject) without any overlapping of images in the test and train databases. Table.1, 2, 3 and 4 shows the result of various illumination normalization techniques with 1 train number of each person and 13 test images of each person with a total number of 100 persons.

Table.1. Face recognition Accuracy in \% using the various illumination normalization techniques 1

\begin{tabular}{|c|c|c|c|c|c|}
\hline $\begin{array}{c}\text { Total No. of } \\
\text { test } \\
\text { Images100 }\end{array}$ & \multicolumn{4}{|c|}{$\begin{array}{c}\text { Face Recognition } \\
\text { using Various Illumination } \\
\text { Normalization techniques } \\
\text { with FKNN Classifier }\end{array}$} \\
\cline { 2 - 6 } $\begin{array}{c}\text { subjects 14 } \\
\text { images }\end{array}$ & $\begin{array}{c}\text { No of Train } \\
\text { images } \\
\text { per subject }\end{array}$ & Log & $\begin{array}{c}\text { Power } \\
\text { Law }\end{array}$ & $\begin{array}{c}\text { Contrast } \\
\text { Stretching }\end{array}$ & HISTEQ \\
\hline $\begin{array}{c}\text { Recognition } \\
\text { Accuracy \% }\end{array}$ & 1 & 20.92 & 30.76 & 52.46 & 52.92 \\
\hline
\end{tabular}

Table.2. Face recognition Accuracy in $\%$ using the various illumination normalization techniques 2

\begin{tabular}{|c|c|c|c|c|c|}
\hline \multirow{2}{*}{$\begin{array}{c}\text { Total No. of } \\
\text { test Images } \\
100 \text { subjects } \\
\text { with } 14 \\
\text { images }\end{array}$} & \multicolumn{5}{|c|}{$\begin{array}{c}\text { Face Recognition } \\
\text { using Various Illumination } \\
\text { Normalization techniques } \\
\text { with FKNN Classifier }\end{array}$} \\
\hline & \begin{tabular}{|c|} 
No of \\
Train \\
images \\
per \\
subject
\end{tabular} & $\begin{array}{c}\text { Adaptive } \\
\text { histogram } \\
\text { equalization }\end{array}$ & $\begin{array}{c}\text { Homomorphic } \\
\text { filter }\end{array}$ & Retinex & $\begin{array}{l}\text { Multiscale } \\
\text { Retinex }\end{array}$ \\
\hline $\begin{array}{l}\text { Recognition } \\
\text { Accuracy \% }\end{array}$ & 1 & 57.07 & 44.53 & 21.30 & 21.15 \\
\hline
\end{tabular}

Table.3. Face recognition Accuracy in \% using the various illumination normalization techniques 3

\begin{tabular}{|c|c|c|c|c|c|}
\hline \multirow{2}{*}{$\begin{array}{c}\text { Total No. of } \\
\text { test Images } \\
1400\end{array}$} & \multicolumn{5}{|c|}{$\begin{array}{c}\text { Face Recognition } \\
\text { using Various Illumination } \\
\text { Normalization techniques } \\
\text { with FKNN Classifier }\end{array}$} \\
\hline & \begin{tabular}{|c|} 
No of \\
Train images \\
per subject
\end{tabular} & DoG & DCTN & Gradient & SQI \\
\hline $\begin{array}{l}\text { Recognition } \\
\text { Accuracy \% }\end{array}$ & 1 & 12.30 & 22.46 & 45.23 & 2.61 \\
\hline
\end{tabular}

Table.4. Face recognition Accuracy in \% using the various illumination normalization techniques 4

\begin{tabular}{|c|c|c|c|}
\hline \multirow{2}{*}{$\begin{array}{l}\text { Total No. of test Images } \\
\text { 100 subjects with 14 images }\end{array}$} & \multicolumn{3}{|c|}{$\begin{array}{c}\text { Face Recognition } \\
\text { using Various Illumination } \\
\text { Normalization techniques } \\
\text { with FKNN Classifier }\end{array}$} \\
\cline { 2 - 4 } & $\begin{array}{c}\text { No of Train } \\
\text { images per subject }\end{array}$ & DCT & DWT \\
\hline Recognition Accuracy \% & 1 & 34.30 & 33 \\
\hline
\end{tabular}

From the above tables, it is found that the adaptive histogram equalization technique gives the highest accuracy \% rate of 57.07 .

The Face recognition accuracy of adaptive histogram equalization technique is tested using various train numbers per person. Table. 5 shows face recognition accuracy of using train numbers 2, 3, 4, 5 and 6 . After increasing the train number from 5 to 6 it is found that the recognition accuracy rate decreases.

Table.5. Face recognition Accuracy in \% using the various illumination normalization techniques 5

\begin{tabular}{|l|c|c|}
\hline \multirow{4}{*}{$\begin{array}{c}\text { Total No. of } \\
\text { test Images }\end{array}$} & \multicolumn{2}{|c|}{$\begin{array}{c}\text { Face Recognition } \\
\text { using Various Illumination } \\
\text { Normalization techniques } \\
\text { with FKNN Classifier }\end{array}$} \\
\cline { 2 - 3 } & $\begin{array}{c}\text { No of Train } \\
\text { images per subject }\end{array}$ & $\begin{array}{c}\text { Adaptive histogram } \\
\text { equalization }\end{array}$ \\
\hline \multirow{3}{*}{$\begin{array}{c}\text { Recognition } \\
\text { Accuracy } \%\end{array}$} & 2 & 58.25 \\
\cline { 2 - 3 } & 3 & 61.63 \\
\cline { 2 - 3 } & 5 & 66.80 \\
\cline { 2 - 3 } & 6 & 72.44 \\
\hline
\end{tabular}

Figure.12 shows the face recognition accuracy rate of all illumination normalization techniques. 


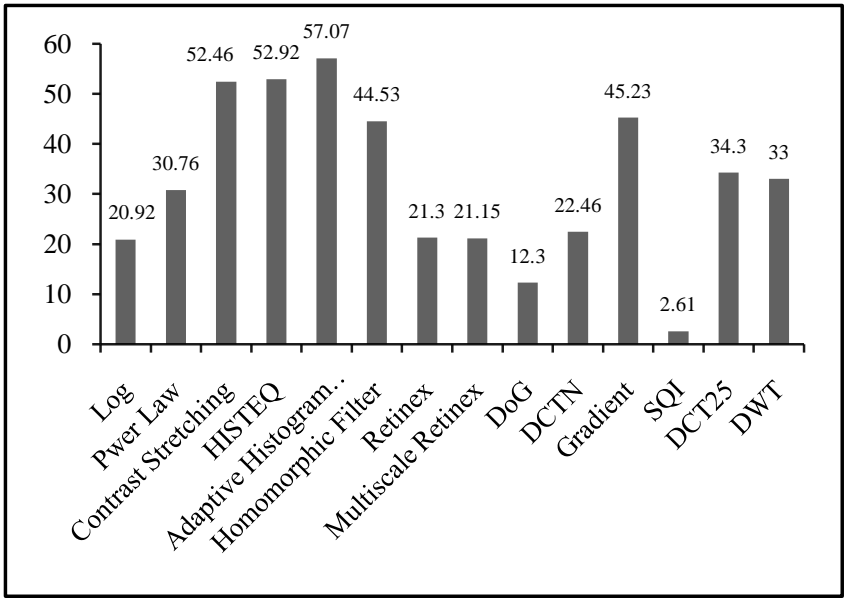

Fig.12. Face recognition accuracy rate of various illumnation normalization techniques

Figure.13 is the plot of number of train images per subject with Recognition accuracy $\%$ in y axis of Adaptive histogram equalization with the highest accuracy rate of $72.44 \%$.

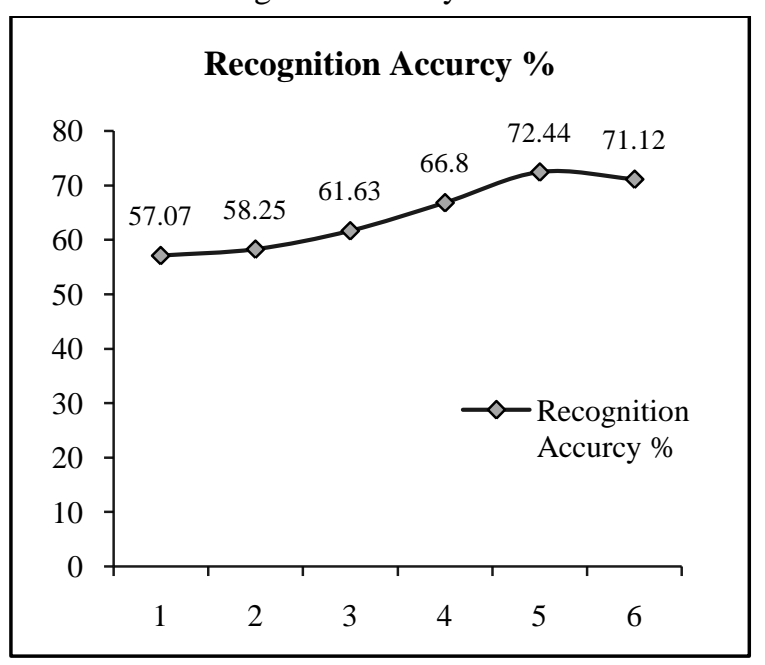

Fig.13. Face Recognition accuracy of Adaptive histogram equalization with different train numbers

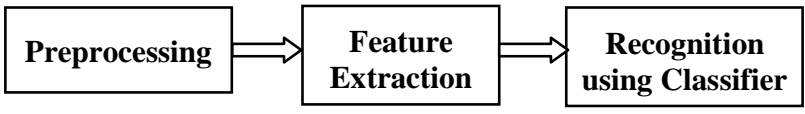

Fig.14. Block Diagram of typical Face recognition system

Figure 14 shows the typical block diagram of face recognition system which consists of three steps preprocessing, feature extraction and recognition using a classifier. In this paper the preprocessing step is done and recognition is done and feature extraction step is not included. After preprocessing the image, if the feature extraction techniques like PCA, LDA, ICA etc. are used then the recognition accuracy would be higher than what is achieved. This work is to identify the better illumination normalization technique without using the feature extraction methods.

\section{CONCLUSION}

Face recognition is a popular biometric authentication since it does not require the aid of the tester like other biometric methods iris, finger print etc. A face image has high dimension. Among the illumination normalization techniques adaptive histogram equalization normalization technique produces highest accuracy rate of $57.07 \%$ with 1 train number for each person and 13 images of same person with a total number of 100 subjects of AR face subset database images. AR database chosen here has varying illumination conditions. However this accuracy is achieved with the simple preprocessing of original face image. When the train numbers is increased up to 5 the recognition accuracy rate achieved is $72.44 \%$ without using any dimensionality reduction techniques like PCA, LDA, ICA, etc.

\section{REFERENCES}

[1] S. Georghiades, P. N. Belhumeur and D. J. Kriegman, "From few to many: Illumination cone models for face recognition under variable lighting and pose", IEEE Transactions on Pattern Analysis and Machine Intelligence, Vol. 23, No. 6, pp. 643-660, 2001.

[2] Thomas Heseltine, Nick Pears, Jim Austin and Zezhi Chen, "Face Recognition: A Comparison of Appearance-Based Approaches", Proceedings of VII ${ }^{\text {th }}$ Digital Image Computing. Techniques and Applications, pp. 59-68, 2003.

[3] Nusrat Ahmed Surobhi and Md. Ruhul, "Employment of Modified Homomorphic filters in Medical Imaging", Daffodil International University Journal of Science and Technology, Vol. 1, No. 1, pp. 60-63, 2006.

[4] Rafael C. Gonzalez and Richard E. Woods, "Digital Image Processing", Addison- Wesley, 1993.

[5] Wen-Hsiung Chen, Smith $\mathrm{C}$ and Fralick S, "A Fast Computational Algorithm for the Discrete Cosine Transform", IEEE Transactions on Communications, Vol. 25, No. 9, pp. 1004-1009, 1977.

[6] T. Zhang, Y.Y. Tang, B. Fang, Z. Shang and X. Liu, "Face recognition under varying illumination using gradient faces", IEEE Transactions on Image Processing, Vol. 18, No. 11, pp. 2599-2606, 2009.

[7] J.M. Keller, M.R. Gray and J.A. Givens, "A fuzzy knearest neighbor algorithm", IEEE Transactions on Systems, Man and Cybernetics, Vol. 15, No. 4, pp. 580585, 1985.

[8] Tamas Kenesei, Balazs Balasko and Janos Abonyi, "A MATLAB Toolbox and its Web based Variant for Fuzzy Cluster Analysis", Proceedings of the $7^{\text {th }}$ International Symposium on Hungarian Researchers on Computational Intelligence, 2006.

[9] Martinez A and Benavente R, "AR Face Database", Technical Report 24, Computer Vision Center, 1998.

[10] Mariusz Leszczyński, "Image Preprocessing for Illumination Invariant Face Verification", Journal of Telecommunications and Information Technology, Vol. 4, No. 4, pp. 19-25, 2010.

[11] Edwin H. Land and John J. McCann, "Lightness and Retinex Theory", Journal of the Optical Society of America, Vol. 61, No. 1, pp. 1-11, 1971. 
[12] W. Chen, M.J. Er and S. Wu, "Illumination compensation and normalization for robust face recognition using discrete cosine transform in logarithmic domain", IEEE Transactions on Systems, Man and Cybernetics, Part B: Cybernetics, Vol. 36, No. 2, pp. 458-466, 2006.

[13] Vitomir Struc, "The INface toolbox v2.1 The Matlab Toolbox for Illumination Invariant Face Recognition Toolbox description and user manual", University of Ljubljana, 2012.

[14] H. Wang, S.Z. Li, Y. Wang and J. Zhang, "Self quotient image for face recognition", Proceedings of the International Conference on Image Processing, Vol. 2, pp. 1397-1400, 2004.
[15] A.H. Mallot, "Computational Vision", The MIT Press, Cambridge, 2000.

[16] Jamie Cook, Vinod Chandran and Sridha Sridharan, "Multiscale representation for 3-D Face recognition", IEEE Transactions on Information Forensics and Security, Vol. 2, No. 3, pp. 529-536, 2007.

[17] N. Ahmed, T. Natarajan and K.R. Rao, "Discrete Cosine Transform", IEEE Transactions on Computers, Vol. C-23, No. 1, pp. 90-93, 1974.

[18] V. Štruc and N. Pavesic, "Performance evaluation of photometric normalization techniques for illumination invariant face recognition", Advances in Face Image Analysis: Techniques and Technologies, pp. 279-300, 2011. 